\title{
Case Report \\ Uncovering Clinical Features of De Novo Philadelphia Positive Myelodysplasia
}

\author{
Aristides Armas, ${ }^{1}$ Chen Chen, ${ }^{2,3}$ Martha Mims, ${ }^{1,2,3,4}$ and Gustavo Rivero ${ }^{1,2,3,4}$ \\ ${ }^{1}$ Baylor St. Luke's Medical Center, Houston, TX 77030, USA \\ ${ }^{2}$ Baylor College of Medicine, Section of Hematology and Oncology, 1 Baylor Plaza, Houston, TX 77030, USA \\ ${ }^{3}$ Department of Pathology, Baylor College of Medicine, 1 Baylor Plaza, Houston, TX 77030, USA \\ ${ }^{4}$ The Dan L. Duncan Comprehensive Cancer Center at Baylor College of Medicine, 1 Baylor Plaza, Houston, TX 77030, USA
}

Correspondence should be addressed to Gustavo Rivero; garivero@bcm.edu

Received 30 November 2016; Accepted 24 January 2017; Published 21 February 2017

Academic Editor: Akimichi Ohsaka

Copyright (c) 2017 Aristides Armas et al. This is an open access article distributed under the Creative Commons Attribution License, which permits unrestricted use, distribution, and reproduction in any medium, provided the original work is properly cited.

Myelodysplastic syndrome (MDS) is cytogenetically heterogeneous and retains variable risk for acute myeloid leukemia transformation. Though not yet fully understood, there is an association between genetic abnormalities and defects in gene expression. The functional role for infrequent cytogenetic alteration remains unclear. An uncommon chromosomic abnormality is the presence of the Philadelphia $(\mathrm{Ph})$ chromosome. Here, we report a patient with $\mathrm{Ph}+\mathrm{MDS}$ treated with low dose Dasatinib who achieved hematologic response for 7 months. In addition, we also examined the English literature on all de novo Ph $+\mathrm{MDS}$ cases between 1996 and 2015 to gain insight into clinical features and outcome.

\section{Case Description}

A 74-year-old male was evaluated for refractory anemia. He had a history of gallbladder cancer treated with radiation (RT) in 2010. His Hb, mean corpuscular volume (MCV), and red cell distribution width (RDW) were $8.3 \mathrm{~g} / \mathrm{dL}, 86.1 \mathrm{fL}$, and $19.8 \%$. White blood cell count, absolute neutrophil count (ANC), and absolute monocyte count (AMC) were $5400 / \mu \mathrm{L}$, $3800 / \mu \mathrm{L}$, and $1080 / \mathrm{uL}$. There was no evidence for basophilia or thrombocytosis. His reticulocyte count, erythropoietin (EPO), and vitamin D level were 0.8\%, 25.8 MIU/ML (2.6$18.5)$, and $76 \mathrm{pg} / \mathrm{mL}$. Ferritin level was $120 \mathrm{ng} / \mathrm{mL}$. Physical exam demonstrated no hepatosplenomegaly. Peripheral blood smear showed normocytic, normochromic anemia with scattered target cells. White blood cells were unremarkable and platelets were adequate. His bone marrow was hypocellular with trilineage dysplasia. His erythroid cells showed nuclear irregularities, budding, and nuclear/cytoplasmic desynchronization (Figures 1(a) and 1(b)). Micromegakaryocytes were hypolobated (Figure 1(c)). Immunohistochemistry revealed no evidence of blasts or monocytosis. Bone marrow findings were consistent with refractory cytopenia with multilineage dysplasia (MDS-MLD) [1]. Karyotype was 46, XY, t (9:22) [4], XY [16] (Figure 1(d)). MDS fluorescence in situ hybridization (FISH) including probes for 5q31, 7q31, 8 centromere, 11q23, and 20q12 was negative. Chromosome microarray analysis (CMA) showed no pathogenic copy number variation (CNV) or copy number neutral loss of heterozygosity (LOH). Next generation gene sequencing demonstrated no relevant leukemia mutations in CSFR1, SF3B1, SRSF2, U2AF1, NRAS, KRAS, FLT3, JAK2, JAK3, DNMT3A, KIT, PHF6, PDGFRA, CDKN2A, IDH1, IDH2, TET2, EZH2, CEBPA, EP300, PTPN11, P53, CREBBP, IKZF1, IKZF3, NOTCH1, RUNX1, $W T 1$, and NPM1. Real time polymerase chain reaction (PCR) for BCR-ABL1 (Major p210 form) was $24.1180 \%$, International Scale (IS). Estimated R-IPSS score was 3 (hemoglobin $[8.3 \mathrm{~g} / \mathrm{dL}]=1$; platelets $[341000 / \mu \mathrm{L}]=0$; absolute neutrophil count $[3800 / \mu \mathrm{L}]=0 ;$ cytogenetic $=2$; blast $=[0 \%]=0)$. Trilineage dysplasia, refractory anemia without basophilia, karyotypic, and molecular clonal evidence for $\mathrm{Ph}+$ disease prompted treatment with Dasatinib at $20 \mathrm{mg}$ orally daily. A low dose was selected given potential risk for worsening cytopenias. His hemoglobin progressively increased by $3 \mathrm{~g} / \mathrm{dL}$ after 12 weeks of treatment (Figure 1(e)). Response was 


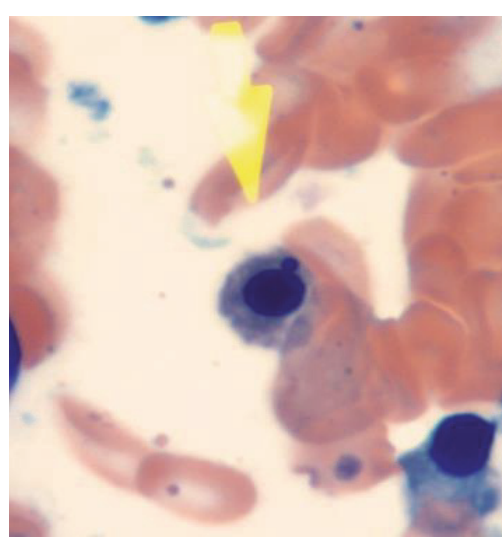

(a)

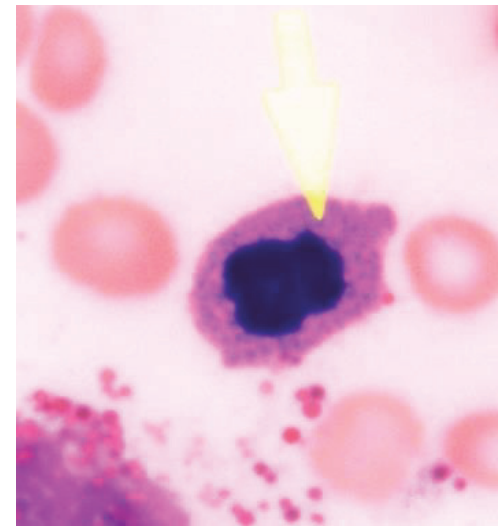

(b)

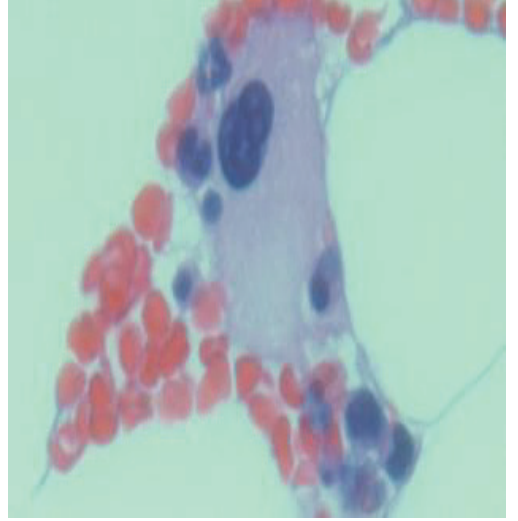

(c)

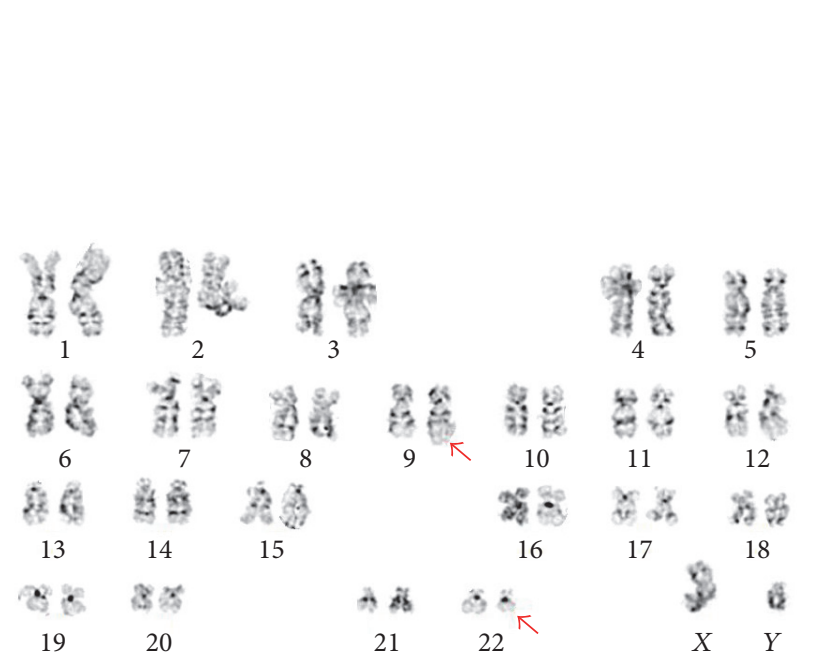

(d)

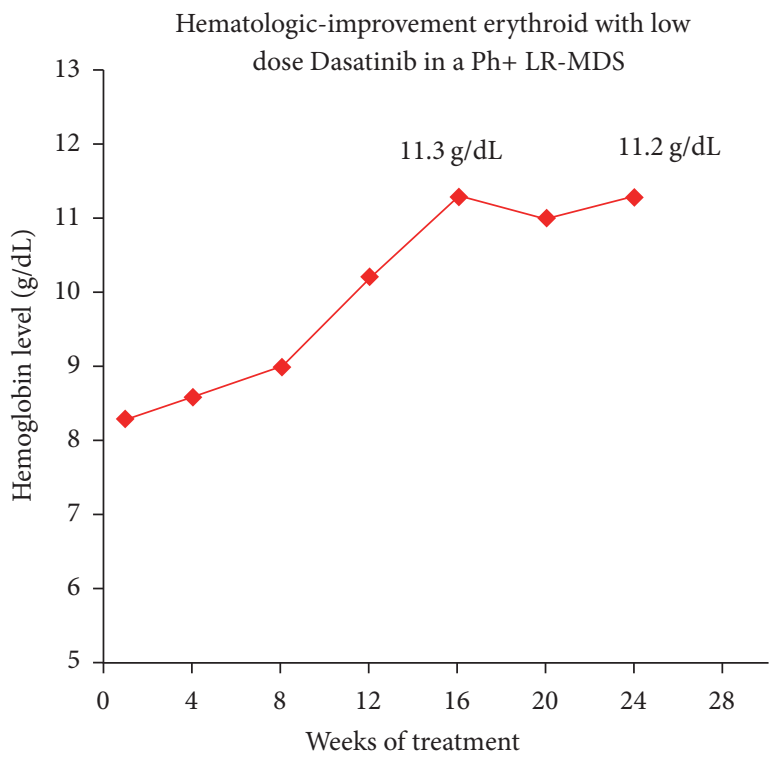

(e)

FIGURE 1: Bone marrow aspirate, biopsy, metaphases cytogenetic, and response to Dasatinib in a Ph+ LR-MDS patient. (a) Erythroid precursor with nuclear irregularity, budding, and nuclear/cytoplasm desynchronization. (b) Dysplastic erythroid precursors with karyorrhexis. (c) shows hypolobated dysplastic megakaryocytes. (d) Patient karyotype showing t $(9 ; 22)$ translocation. (e) Progressive increase in hemoglobin level for a Ph + LR-MDS [RCMD] patient treated with low-dose Dasatinib. Progressive H-E was observed at 12 weeks. HI-E was sustained at 24 weeks of treatment.

maintained for a total of 24 weeks (Figure 1(e)). BCR-ABL1 transcripts were undetectable $(0.0006 \%$, IS) after 4 months of treatment. After 7 months of follow-up, patient opted for hospice care after developing pneumonia that led to respiratory failure.

\section{Methods}

In addition to our case, 9 patients diagnosed with de novo $\mathrm{Ph}+\mathrm{MDS}$ were identified from PubMed search [28]. De novo Ph+ MDS was defined as unequivocal morphologic evidence for myeloid dysplasia and initial karyotype examination positive for $\mathrm{Ph}$ abnormality. Given potential biologic confounders, treatment related MDS, CMML, and $\mathrm{MDS} / \mathrm{MPN} \mathrm{Ph}+$ cases were excluded. In our 10 patients' cohort, we investigated world health organization (WHO) 2008 classification, demographics, histopathologic bone marrow findings, and clinical outcome. Our primary aim was to identify relevant disease features that could facilitate disease management.

\section{Cohort Analysis}

Patient's characteristics and cytogenetic abnormalities are depicted in Table 1. Most of patients were males 6/10 (60\%). Median age at diagnosis was 66 years (range, $49-74)$. According to WHO 2008, 3/7 (43\%) and 2/7 (29\%) patients were RAEB-2 and RCMD, respectively. RAEB-1 and RCUD (1 each) represented two additional patients with morphologic available data. Median hemoglobin, platelet, white, and blast 


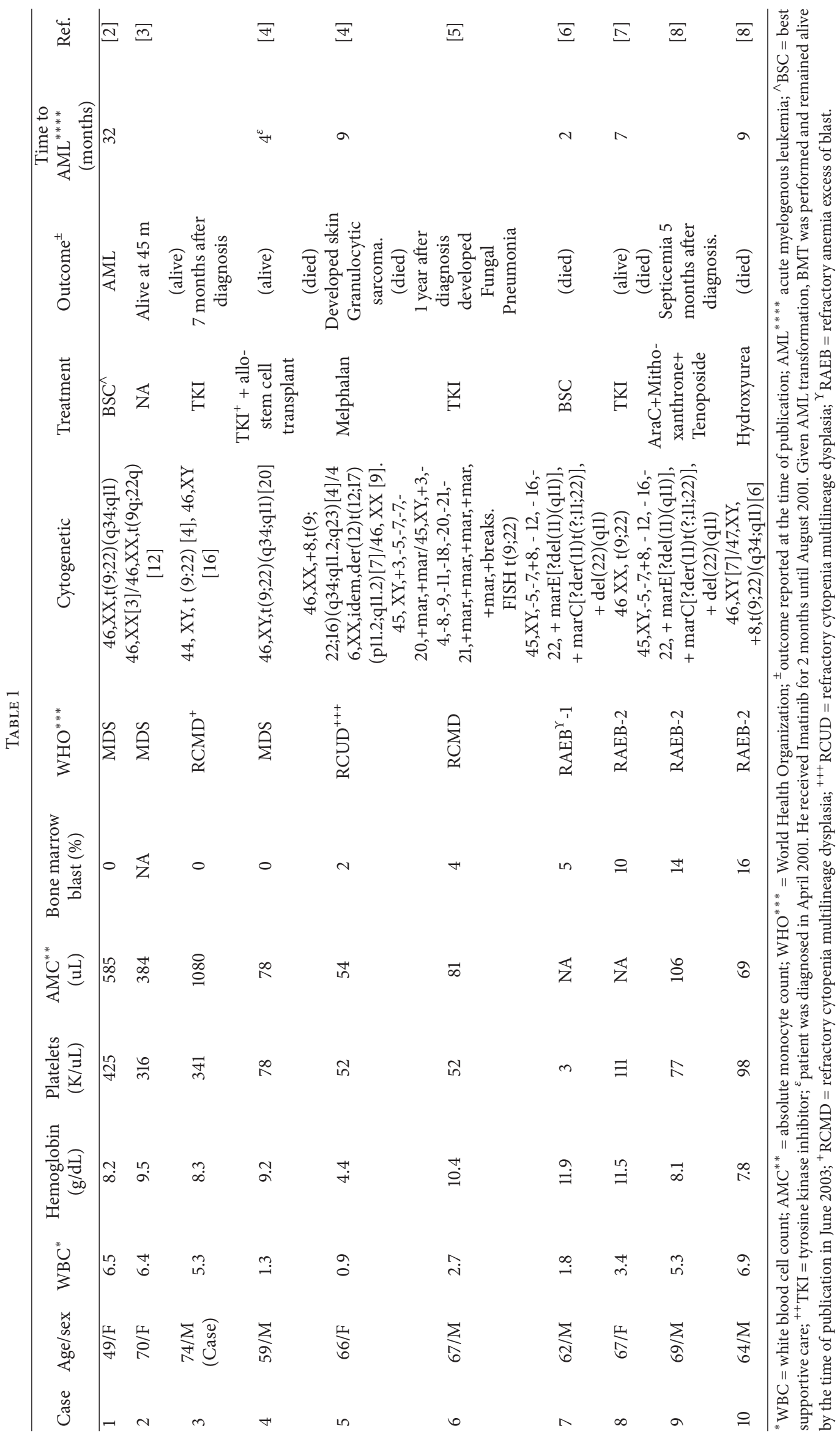


count were $8.75 \mathrm{~g} / \mathrm{dL}, 88 \mathrm{~K} / \mathrm{uL}, 4.35 \mathrm{~K} / \mathrm{uL}$, and $4 \%$. Among 10 patients, $\mathrm{Ph}$ translocation, as a sole chromosomic abnormality, was detected in 5/10 (50\%). Trisomy 8 occurred commonly as an additional karyotypic alteration in $4 / 10$ (40\%) of patients and was frequently associated with complex cytogenetic spanning monosomies 5 and 7. TKI treatment resulted in hematologic remission in 4 patients leading to a median response duration of 7 months. In 5 patients with available follow-up, median time to AML transformation was 7 months. Interestingly, in patients with de novo $\mathrm{Ph}+\mathrm{MDS}$, a platelet count of more or less than $100 \mathrm{~K} / \mathrm{uL}$ resulted in an overall survival (OS) of 32 versus 9 months.

\section{Discussion}

MDS is cytogenetically heterogeneous presenting with karyotypic abnormalities in about $50 \%$ of patients. Chromosomic gains and, most commonly, somatic loss of tumor suppressor genes (TSG) by structural and epiphenomenon's modifications result in impaired differentiation [9]. Translocation (9; 22) is frequently observed in chronic myelogenous leukemia (CML). The 2008 world health organization (WHO) and the 2016 revision for classification of myeloid neoplasm emphasizes that CML BCR-ABLl ${ }^{+}$phenotype must include significant myeloproliferation. A distinction for chronic myelomonocytic leukemia (CMML) is the prerequisite for persistent monocytosis in peripheral blood $\geq 1000^{9} / \mathrm{L}[1,10]$. Paradoxically, the translocation $(9 ; 22)$ is rarely associated with dysplastic hematopoiesis suggesting that the abnormality may have antagonistic disease-initiating role. In this report, we describe a $\mathrm{Ph}+\mathrm{MDS}$ patient exhibiting refractory anemia and bone marrow trilineage dysplasia that attained HI-E after low dose Dasatinib. Lack of peripheral blood and bone marrow proliferation favored clonal disorder unrelated to myeloproliferative neoplasm. The presence of $\mathrm{Ph}+$ metaphases and low-grade BCR-ABL transcripts suggest that his MDS was, at least in part, resultant from aberrant kinase overexpression. Given the rare nature of de novo $\mathrm{Ph}+\mathrm{MDS}$, we selected 9 additional cases to evaluate disease presentation and outcome. Previously, Keung et al. described three de novo $\mathrm{Ph}+\mathrm{MDS}$ cases and reviewed 18 additional patients [4]. However, contrasting with our de novo cohort, his study included $\mathrm{Ph}+$ treatment-related disease and CMML cases. In addition, about $48 \%$ of patients lacked $\mathrm{Ph}+$ chromosomic abnormality and rather acquired the translocation sequentially at disease progression. It is possible that Keung et al. study design limits our ability to characterize disease initiating mechanisms and de novo disease outcome. We observed that $50 \%$ of patients present with $\mathrm{t}(9 ; 22)$ as a sole abnormality. However, typical MDS chromosomic abnormalities, such as +8 , frequently coexist in complex $\mathrm{Ph}+$ metaphases. The disease exhibits high-risk features characterized by complex karyotype and elevated proportion of blast and thrombocytopenia. Previous studies have demonstrated that thrombocytopenia adversely impacts MDS outcome [11]. Neukirchen et al. specifically highlights that patients presenting with platelet count lower than $100000 / \mathrm{uL}$ retained inferior outcome [12]. It is possible that $\mathrm{Ph}+\mathrm{MDS}$ exhibits similar prognostic variables as those observed in Ph negative MDS. A short median time to AML transformation of 7 months suggests that $\mathrm{Ph}+\mathrm{MDS}$ is a highly aggressive disease, especially if it is associated with complex karyotype and thrombocytopenia. In Keung et al. study [4], median time progression to AML was reported as 13 months. This most favorable prognosis may be biased by inclusion of indolent $\mathrm{Ph}+$ myeloproliferative disorders.

Three $\mathrm{Ph}+\mathrm{MDS}$ patients developed isolated refractory anemia, normal platelets, low blast count, and more favorable outcome. This led us to hypothesize that their IE functionally originates from similar $\mathrm{Ph}$ negative low risk MDS mechanisms. Given potential BCR-ABL disease initiating role, TKI represents an attractive intervention. However, to date, the efficacy of this therapy on $\mathrm{Ph}+\mathrm{MDS}$ remains poorly characterized. In our cohort study, TKI treatment resulted in about 7 months' response in 4 patients. Similarly, TKI was feasible in our reported case demonstrating a correlation between HI-E and molecular remission. Interestingly, low dose of Dasatinib led to limited side effects. IE is associated with abnormal paracrine/autocrine pathways leading to hemopoietic failure; specifically elevated IL- $1 \beta$, TNF- $\alpha$, and INF- $\gamma$ levels are central to low risk-MDS (LR-MDS) [13]. In recent years, the role of deregulated innate immunity has emerged as potential etiology for LR-MDS. Although our patient's HI-E could be attributed to Dasatinib induced BCR-ABL inhibition, it is possible that an "off-target" Dasatinib effect on deregulated cytokines facilitated IE improvement. In vitro studies have demonstrated that Dasatinib suppresses the production of IL- 6 and TNF $\alpha$ and favors production of anti-inflammatory cytokines, such as IL-10 in primary macrophages [14]. In addition, Src-family kinases (SFK), a potential Dasatinib target, participates in toll-like receptor-2 (TLR-2) mediated NF$\mathrm{kB}$ activation leading to elevated IL- 6 and TNF $\alpha$ levels [15]. Recent reports link TLR-2 deregulation and MDS pathogenesis [16]. Dasatinib may potentially cooperate to normalize IL- 6 and TNF $\alpha$ levels inappropriately elevated in LR-MDS.

There are limitations to our study. The retrospective nature of the study could have biased our survival estimation for patients with high and low risk features given inaccurate censoring at the time of publication. Concerning our case, the lack of posttreatment bone marrow evaluation limits our ability to assign a direct favorable TKI effect to improve dysplastic marrow features. However, low dose Dasatinib led to molecular remission and improved IE. This may suggest a favorable drug effect on $\mathrm{Ph}+\mathrm{MDS}$ disease initiating mechanisms and chronic marrow inflammation. To date, our study is the first to investigate a cohort of newly diagnosed $\mathrm{Ph}+\mathrm{MDS}$. The disease potentially recapitulates low and high risk features observed in $\mathrm{Ph}$ negative disease. It is likely that high risk $\mathrm{Ph}+\mathrm{MDS}$ could exhibit short latency to AML necessitating early chemotherapy and even allo-stemcell transplantation. Future directions should evaluate the role and potential mechanism of action of TKIs in Ph + MDS patients presenting with low risk features.

\section{Competing Interests}

All authors reported no competing interests. 


\section{Authors' Contributions}

Dr. Armas, Mims, and Rivero contributed equally to manuscript writing. Dr. Chen provided hemopathologic description for the case. Dr. Rivero designed plan for investigation and report of the case.

\section{References}

[1] D. A. Arber, A. Orazi, R. Hasserjian et al., "The 2016 revision to the World Health Organization classification of myeloid neoplasms and acute leukemia," Blood, vol. 127, no. 20, pp. 23912405, 2016.

[2] D. G. Roth, C. M. Richman, and J. D. Rowley, "Chronic myelodysplastic syndrome (preleukemia) with the Philadelphia chromosome," Blood, vol. 56, no. 2, pp. 262-264, 1980.

[3] A. Berrebi, R. Bruck, M. Shtalrid, and J. Chemke, "Philadelphia chromosome in idiopathic acquired sideroblastic anemia," Acta Haematologica, vol. 72, no. 5, pp. 343-345, 1984.

[4] Y.-K. Keung, M. Beaty, B. L. Powell, I. Molnar, D. Buss, and M. Pettenati, "Philadelphia chromosome positive myelodysplastic syndrome and acute myeloid leukemia-retrospective study and review of literature," Leukemia Research, vol. 28, no. 6, pp. 579-586, 2004.

[5] S. Dutta, P. Kumari, N. Ks et al., "Philadelphia chromosomepositive myelodysplastic syndrome: is it a distinct entity?" Acta Haematologica, vol. 129, no. 4, pp. 215-217, 2013.

[6] J. H. Ohyashiki, K. Ohyashiki, H. Fujieda et al., "Myelodysplastic syndrome with Philadelphia-like chromosome without bcr rearrangement," Cancer Genetics and Cytogenetics, vol. 35, no. 2, pp. 151-158, 1988.

[7] M. W. Drummond, C. J. Lush, M. A. Vickers, F. M. Reid, J. Kaeda, and T. L. Holyoake, "Imatinib mesylate-induced molecular remission of Philadelphia chromosome-positive myelodysplastic syndrome," Leukemia, vol. 17, no. 2, pp. 463-465, 2003.

[8] J. F. Lesesve, X. Troussard, C. Bastard et al., "p190(bcr/abl) rearrangement in myelodysplastic syndromes: two reports and review of the literature," British Journal of Haematology, vol. 95, no. 2, pp. 372-375, 1996.

[9] G. Garcia-Manero, "Myelodysplastic syndromes: 2011 update on diagnosis, risk-stratification, and management," American Journal of Hematology, vol. 86, no. 6, pp. 490-498, 2011.

[10] J. W. Vardiman, J. Thiele, D. A. Arber et al., "The 2008 revision of the World Health Organization (WHO) classification of myeloid neoplasms and acute leukemia: rationale and important changes," Blood, vol. 114, no. 5, pp. 937-951, 2009.

[11] H. Kantarjian, F. Giles, A. List et al., "The incidence and impact of thrombocytopenia in myelodysplastic syndromes," Cancer, vol. 109, no. 9, pp. 1705-1714, 2007.

[12] J. Neukirchen, S. Blum, A. Kuendgen et al., "Platelet counts and haemorrhagic diathesis in patients with myelodysplastic syndromes," European Journal of Haematology, vol. 83, no. 5, pp. 477-482, 2009.

[13] S. M. Kornblau, D. McCue, N. Singh, W. Chen, Z. Estrov, and K. R. Coombes, "Recurrent expression signatures of cytokines and chemokines are present and are independently prognostic in acute myelogenous leukemia and myelodysplasia," Blood, vol. 116, no. 20, pp. 4251-4261, 2010.

[14] J. Ozanne, A. R. Prescott, and K. Clark, "The clinically approved drugs dasatinib and bosutinib induce anti-inflammatory macrophages by inhibiting the salt-inducible kinases," Biochemical Journal, vol. 465, pp. 271-279, 2015.
[15] J. Toubiana, A.-L. Rossi, N. Belaidouni et al., "Src-familytyrosine kinase Lyn is critical for TLR2-mediated NF- $\kappa$ activation through the PI 3-kinase signaling pathway," Innate Immunity, vol. 21, no. 7, pp. 685-697, 2015.

[16] Y. Wei, S. Dimicoli, C. Bueso-Ramos et al., "Toll-like receptor alterations in myelodysplastic syndrome," Leukemia, vol. 27, no. 9, pp. 1832-1840, 2013. 


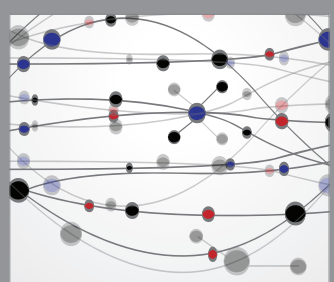

The Scientific World Journal
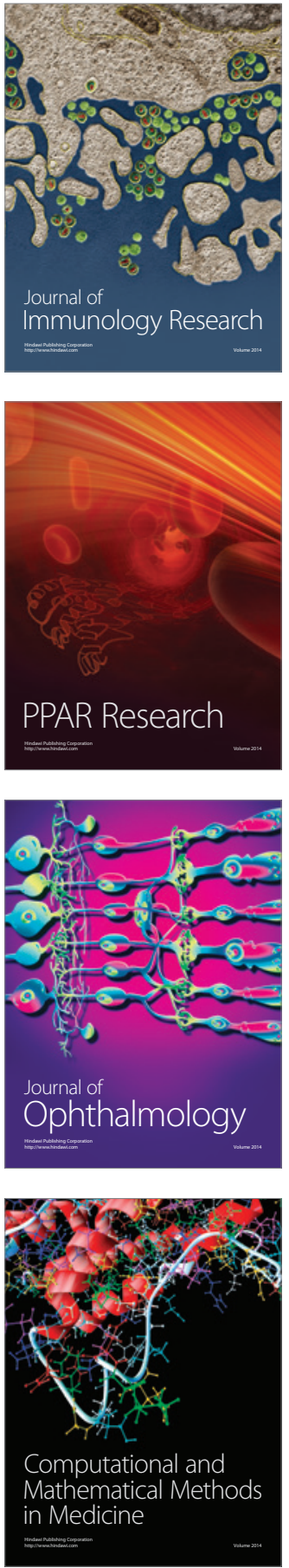

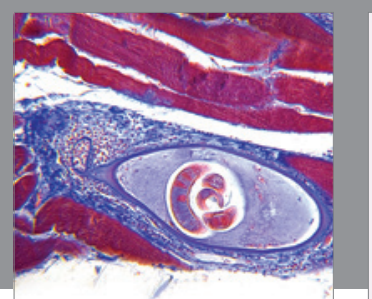

Gastroenterology Research and Practice
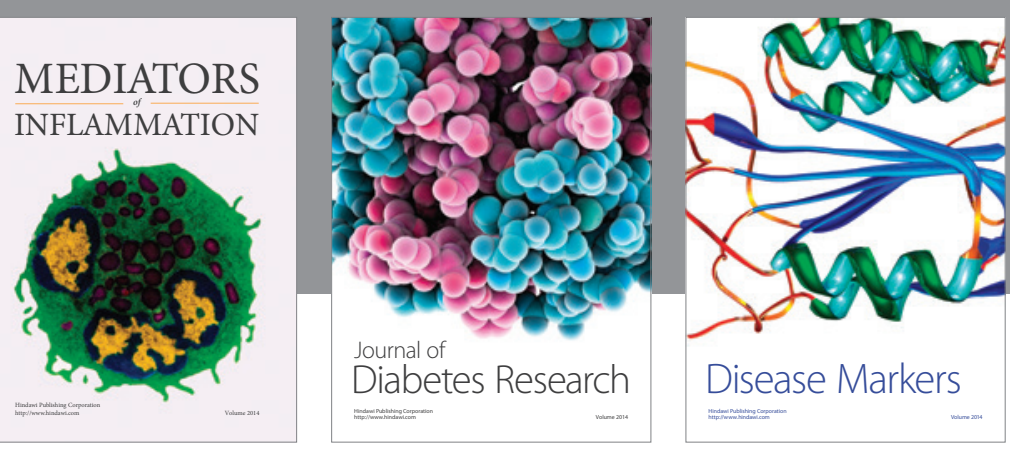

Disease Markers

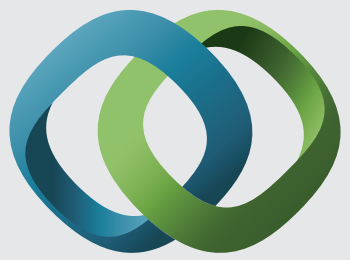

\section{Hindawi}

Submit your manuscripts at

https://www.hindawi.com
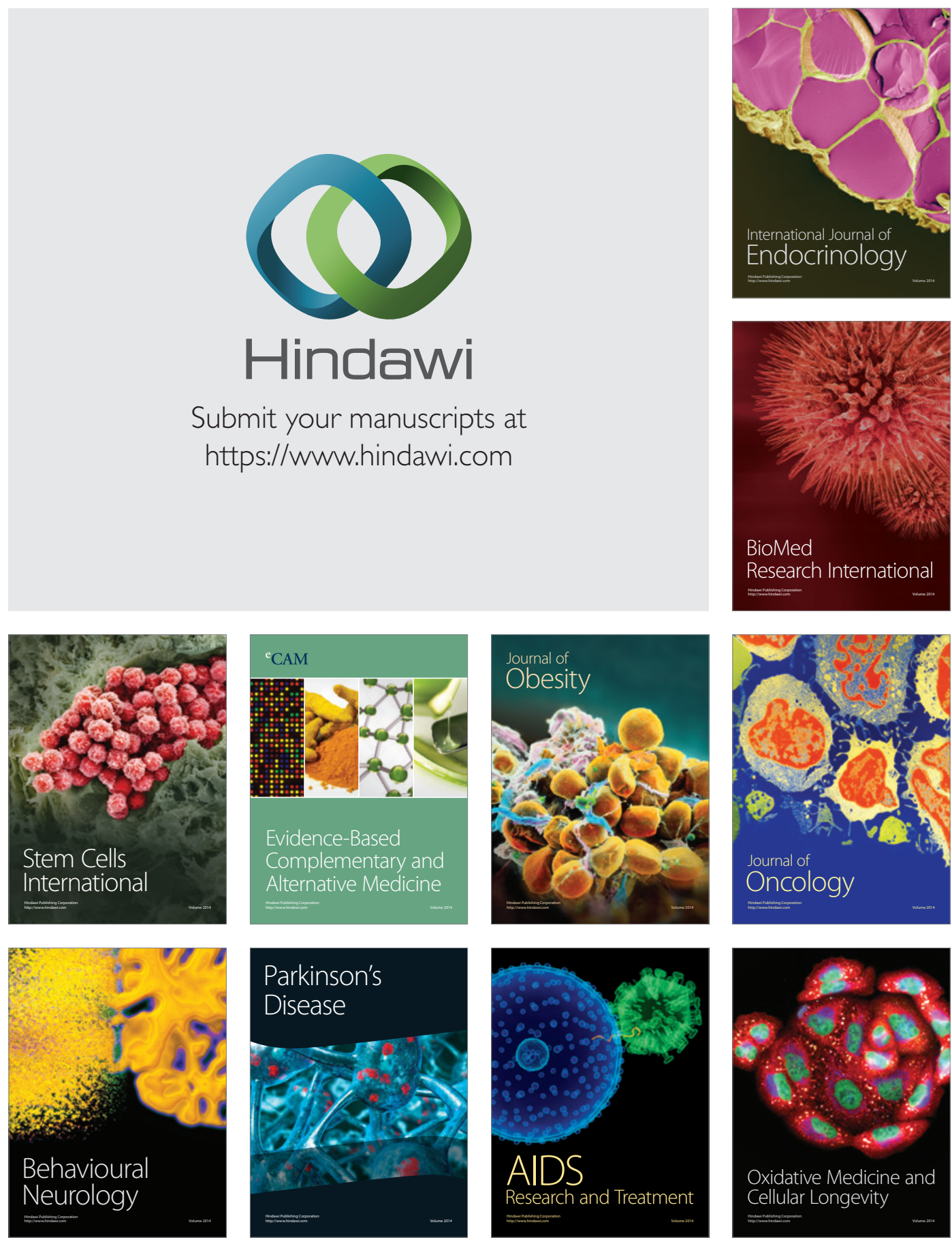